\title{
Proposal of a super-symmetric microcalorimeter for single-step realization of an RF primary power standard
}

\author{
Emil Vremera ${ }^{1}$, Luciano Brunetti ${ }^{2}$ \\ 1 "Gheorghe Asachi" Technical University of lasi, Faculty of Electrical Engineering, Bld. Dimitrie Mangeron 21-23, 700050, lasi, Romania \\ ${ }^{2}$ Istituto Nazionale di Ricerca Metrologica - INRiM, Strada delle Cacce 91, 10135 Torino, Italia
}

\begin{abstract}
The realization of RF primary power standards by means of the microcalorimeter technique requires a long measurement time due to the necessity of performing two completely distinct steps: measurement of the power standard and system calibration. Such a procedure is detrimental to overall measurement accuracy; therefore, in this paper, a new improved design of a dual-channel broadband twin microcalorimeter is proposed. It is based on modified hardware and new, more suitable algorithms, which allows for the selfcalibration of the system without the dismounting/mounting operations between the measurement cycles. The paper has some key results: a significantly shorter measurement time, maintenance of (at least) the usual microcalorimeter accuracy, an improvement in thermal noise rejection, perfect correlation of the measured data series, and direct calculus by a simple formula.
\end{abstract}

\section{Section: RESEARCH PAPER}

Keywords: RF power standard; coaxial microcalorimeter; super-symmetric inset, thermoelectric sensor, insulating RF-transmission line

Citation: Emil Vremera, Luciano Brunetti, Proposal of a super-symmetric microcalorimeter for single-step realization of an RF primary power standard, Acta IMEKO, vol. 7, no. 4, article 3, December 2018, identifier: IMEKO-ACTA-07 (2018)-04-03

Editor: Vilmos Pálfi, Budapest University of Technology and Economics, Hungary

Received March 23, 2018; In final form October 2, 2018; Published December 2018

Copyright: (C) 2018 IMEKO. This is an open-access article distributed under the terms of the Creative Commons Attribution 3.0 License, which permits unrestricted use, distribution, and reproduction in any medium, provided the original author and source are credited

Corresponding author: Emil Vremera, e-mail: evremera@tuiasi.ro

\section{INTRODUCTION}

A microcalorimeter is a measurement system that is specifically used for determining the effective efficiency of the power sensors at small power levels $(1-10 \mathrm{~mW})$. Such a system is operative in the most important National Metrology Institutes (NMIs), which implement different architectures with the aims of obtaining a high level of accuracy, wide bandwidth, and limited measurement time.

Basically, this system may be seen either on a thermostatic water bath or an active thermostatic shield, which creates a highly stable thermal environment for single or, more often, twin thermal loads. Figure 1 shows a scheme of a typical twin structure in coaxial transmission lines.

The manner in which this system works has been widely discussed in the relevant literature by other researchers [1-3] and by us [4-6]. However, independent of the technical solution adopted in each laboratory, the effective efficiency of the power sensor used as the calorimetric load can be expressed as the rational function of sensor mount temperatures. These ones are measured with a differential thermometer in two states of thermal equilibrium, obtained by the supply (or not) of the measurement channel with radio-frequency $(\mathrm{RF})$ power.
The critical points of the microcalorimeters are: the temperature stability of the thermostatic shields, the degree of symmetry of the twin inset (if this configuration is used), and most importantly, the dismounting/mounting operations

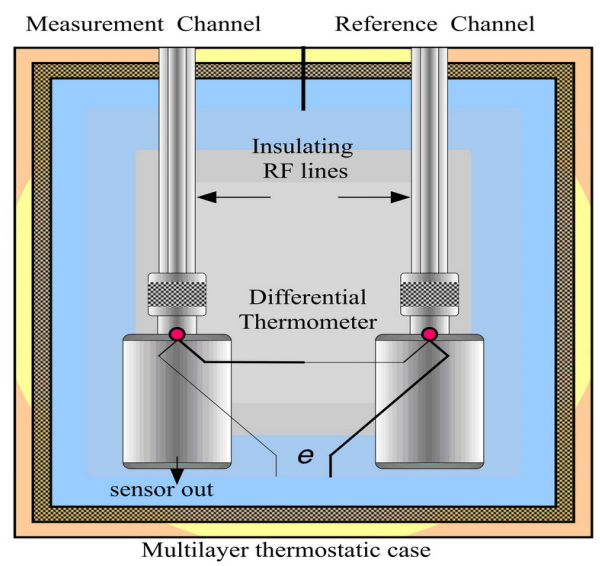

Figure 1 . Scheme of a classic coaxial microcalorimeter based on the twin architecture. 
requested by the microcalorimeter calibration step. This latter operation has an effect on non-coherent datasets, acquired during the microcalorimeter calibration and in the power sensor measurements. Another disadvantage of such microcalorimeters is that they run for a long time due to the high time constant and the large number of test frequencies that are usually requested.

\section{ACTUAL STAGE}

Some NMIs, e.g., Physikalisch Technische Bundesanstalt (PTB), Istituto Nazionale di Ricerca Metrologica (INRiM), and the Korean Research Institute of Standards and Science (KRISS), implement the RF power standard mainly by using thermoelectric power sensors. Other NMIs, such as the National Institute of Standards \& Technology in the US (NIST) and the National Institute of Advanced Industrial Science and Technology in Japan (AIST), prefer bolometric power sensors. In any case, progress in this field is possible if suitable choices are made for each of the following technical options:

twin loads or a single load;

compensations or corrections;

direct results or postponed calculations;

mid-term or long-term measurements;

power-switching (or not) between channels in the twin architecture;

thermal conduction or thermal insulation;

an inner thermal shunt or high inner thermal resistance; thermocouples or bolometers as thermal loads.

Unfortunately, not all of the previously listed points have been thoroughly considered by the scientific community until now, especially those concerning the dry twin-microcalorimeter for thermoelectric loads.

\section{SINGLE-STEP MEASUREMENT PROPOSAL}

In order to measure the power sensors' effective efficiency (which depends on frequency) with the microcalorimeter, two different measurement steps are necessary. In the first step, the power sensor under test is fed alternately with the RF power and the reference power, respectively, and a raw effective efficiency of the power sensor is calculated [4].The second step is necessary to make corrections to the contribution of the microcalorimeter feeding lines. Unfortunately, several problems arise in the calibration step, including microcalorimeter dismount/mount operations, long-term thermal equilibrium, inherent uncontrollable changes in the thermal paths, differences between the power levels adjusted for each step, and very low measured values [1-5].

The power-switching method, proposed in [6] by us, allows us to obtain the final result without corrections or microcalorimeter dismounting/mounting operations, but the involved quantities are, however, obtained in different measurement phases and by changing the instrumentation setup. This contributes to the generation of non-coherent datasets and, therefore, possibly undetected measurement errors.

Only with a new design of the feeding line complex can the classic twin microcalorimeter [4-5] allow single-step measurements and, therefore, direct results for the power sensors' effective efficiency. The technical solution that we propose involves duplicating the classic twin-line inset [4] according to the scheme in Figure 2 [7] in order to obtain the needed super-symmetric inset. Such a proposal was initially imagined as a solution to the intrinsic failure of the classic twin structure in creating a reference channel whose thermal behavior towards the external environment should be the same as the measurement channel. Because this does not happen in practice due to the small but unavoidable differences in the thermal paths, rejection of the thermal noise of the external environment is not yet satisfactory in terms of obtaining the best measurement accuracy. However, the new architecture, which we have named the super-symmetric microcalorimeter, has turned out to be useful in realizing the measurement process for the effective efficiency parameter in a single step. This proposal requires changes to the microcalorimeter design, the instrumentation setup, and measurement algorithms.

a)
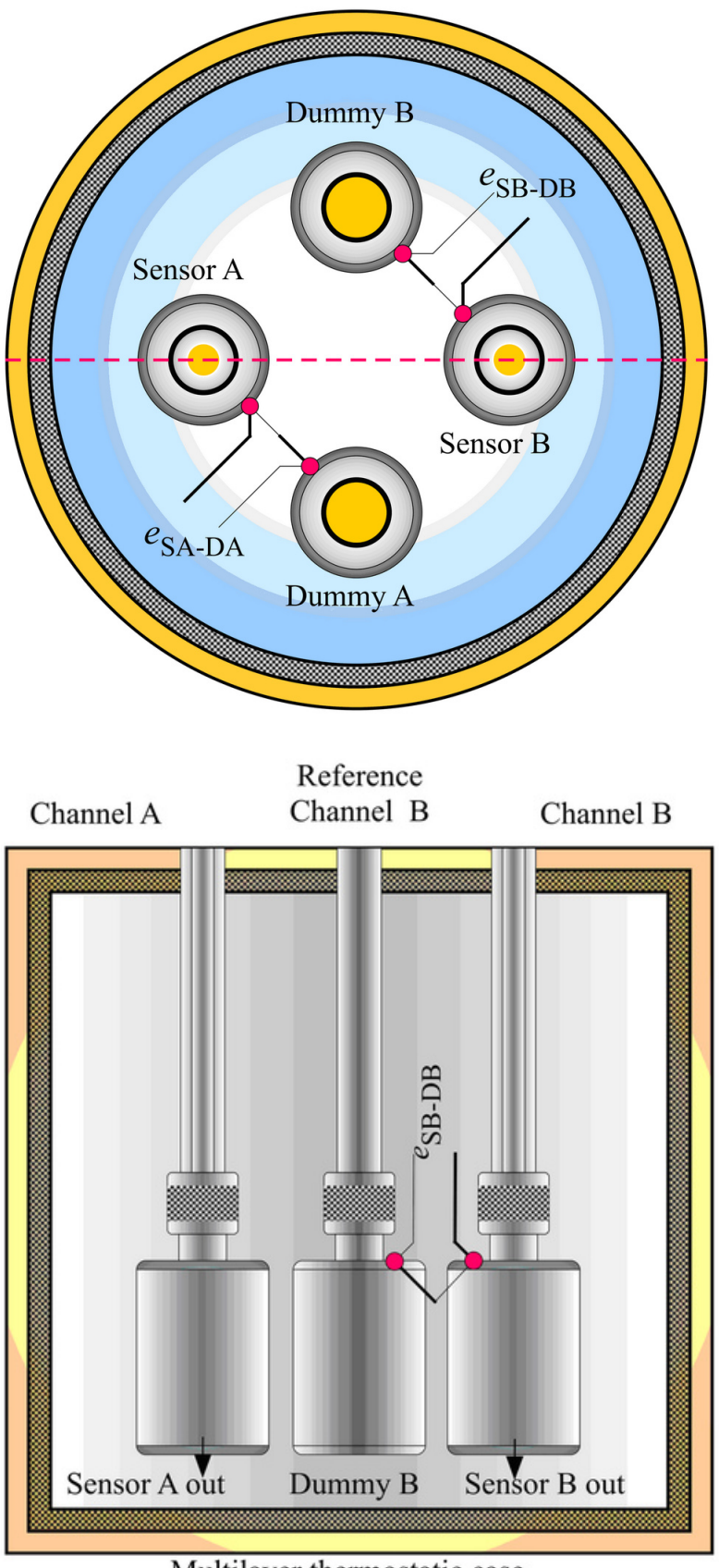

b)

Multilayer thermostatic case

Figure 2. a) Scheme of a cross-section of the new coaxial microcalorimeter inset at the base of power sensor input connector, where thermometers measure the temperature differences; b) Schematic vertical cross-section of the new microcalorimeter. 


\section{NEW TWIN-LOAD MICROCALORIMETER}

At each of channels A and B in the classic twin configuration [5], also shown in Figure 1, an additional transmission line is coupled so that both A and B have their own thermal reference channel. Every additional thermal reference channel is terminated with a dummy load, which, from the electromagnetic point of view, acts as a broadband low loss short circuit. Figure 2 shows two schematics of the axial and transverse sections of the new coaxial microcalorimeter. The channels A and B are fed, alternately, by the RF power and by the reference power, whereas the new reference channels are fed only with the RF power. This scheme must compensate for RF losses in the power sensors' feeding lines and further reduce the effects of the external environment on the measures. In this manner, we can obtain a virtual isothermal reference plane at the sensors' input connectors level as in the case of a lossless ideal feeding line.

In practice, each measurement channel must be electrically balanced against its own reference channel to compensate for any imperfections in the device realizations. A simple balance circuit may be introduced like that which is shown in Figure 3.

\subsection{DESCRIPTION OF SYSTEM COMPONENTS}

The most important components of a microcalorimeter are described in detail as follows:

A) The feeding lines are surely the most critical component of the system because they introduce the dominant error term in the whole measurement process. The general criteria for a suitable design for the feeding lines include that it must match both thermal and electrical behaviors very well and it must have good and adjustable thermal insulation, an inner thermal shunt present at the reference plane level, and a small reflection coefficient at the test load input port. A new profiled machinable air-gap insulating line, as shown in Figure 4, is proposed in order to fulfill these goals. The device is different than that which is described in [8] and incorporates further improvements. Concerning the effect of a $1 \mathrm{~mm}$ air gap, we point out that an improvement of about $50 \%$ of the thermal insulation $[9,10]$ is obtained and does not have notable changes in the S-parameter values in the entire working bandwidth for a $2.92 \mathrm{~mm}$ coaxial line, as Table 1 shows. A particular feature of this new insulation

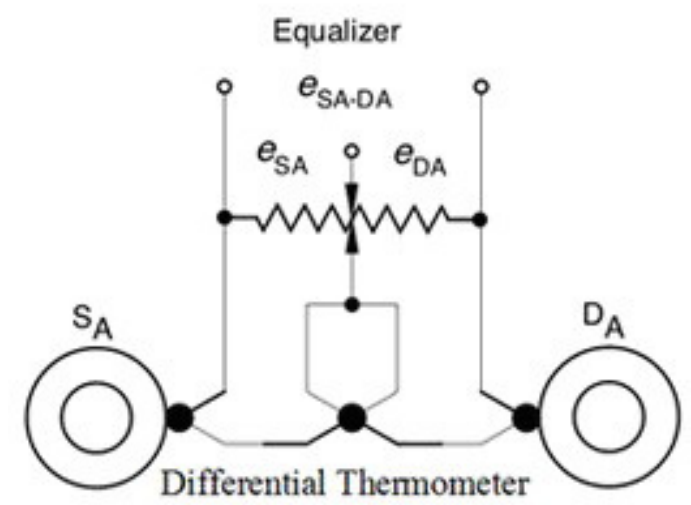

Figure 3. Scheme used to eliminate the electrical dissymmetry between measurement and reference channels.
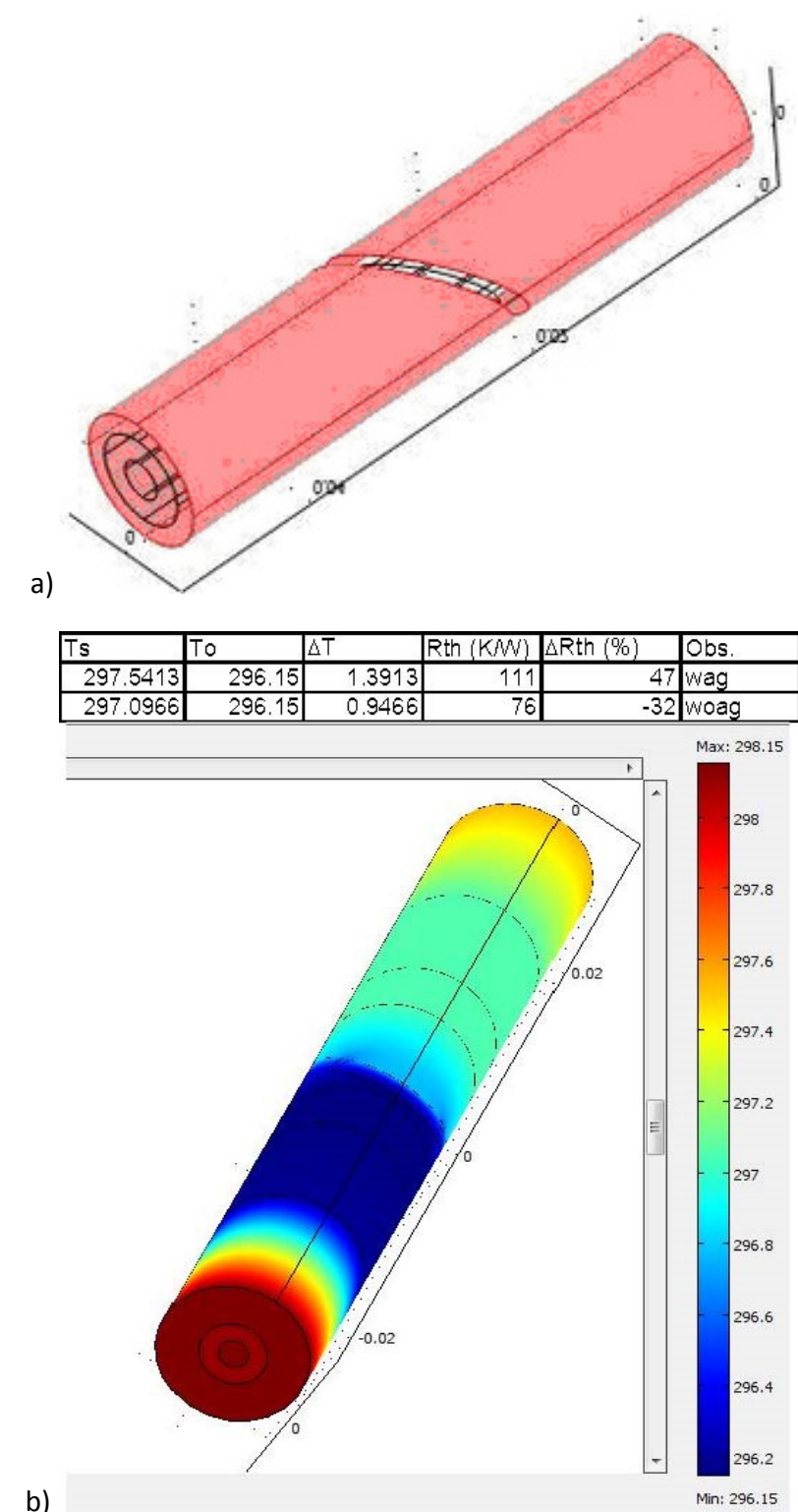

b)

Figure 4. a) Profiled machinable air-gap insulating line. b) Improvements in the thermal isolation (COMSOL Multiphysics).

$T o=$ temperature at the thermal reference plane; $T \mathrm{~s}=$ temperature at the input power cross-section; $R$ th = thermal resistance; wag = with air gap; woag = without air gap; inner-line thermal shunt, present [11].

line is that, apart from the $45^{\circ}$ angle of the air gap cross-section and four direct current (DC) thin wall small bridges, there is also an internal thermal shunt $[11,14]$.

B) The dummy loads are also important. They must be good "imitations" of the power sensors that are undergoing calibration in terms of their mechanical size and thermal properties [1]. A dummy load must be fitted with a metrological-grade RF connector and behaves as a short circuit as much as possible. The sensors' output wires must also be replicated and electrically connected in the same manner for reproducing the same thermal paths and bridges as the real sensors in the test case.

C) The microcalorimeter thermal sensing devices consist of two differential thermopiles with two balanced asymmetric outputs of the same polarity. They directly measure the thermal load response, both absolute and relative to its thermal reference load, for each channel, as shown in Figure 2 and Figure 3. 
Table 1. Scattering parameter trend versus frequency for the insulating section with and without air gap; inner line thermal shunt was present [11].

\begin{tabular}{|c|c|c|c|}
\hline Parameter & Value & Type of IL & Frequency \\
\hline $\mathrm{S}_{21}$ & 0.9900 & wag & $1 \mathrm{GHz}$ \\
\hline $\mathrm{S}_{21}$ & 0.9901 & wag & $20 \mathrm{GHz}$ \\
\hline $\mathrm{S}_{21}$ & 0.9875 & wag & $40 \mathrm{GHz}$ \\
\hline $\mathrm{S}_{21}$ & 0.9885 & woag & $40 \mathrm{GHz}$ \\
\hline $\mathrm{S}_{11}$ & 0.0297 & wag & $1 \mathrm{GHz}$ \\
\hline $\mathrm{S}_{11}$ & 0.0186 & wag & $20 \mathrm{GHz}$ \\
\hline$S_{11}$ & 0.0686 & wag & $40 \mathrm{GHz}$ \\
\hline $\mathrm{S}_{11}$ & 0.0687 & woag & $40 \mathrm{GHz}$ \\
\hline \multicolumn{4}{|c|}{ Lumped ports situation } \\
\hline $\mathrm{S}_{21}$ & 1.0028 & wag & $1 \mathrm{GHz}$ \\
\hline $\mathrm{S}_{21}$ & 1.0022 & wag & $20 \mathrm{GHz}$ \\
\hline$S_{21}$ & 0.9992 & wag & $40 \mathrm{GHz}$ \\
\hline $\mathrm{S}_{21}$ & 0.9960 & woag & $40 \mathrm{GHz}$ \\
\hline$S_{11}$ & 0.0205 & wag & $1 \mathrm{GHz}$ \\
\hline $\mathrm{S}_{11}$ & 0.0190 & wag & $20 \mathrm{GHz}$ \\
\hline $\mathrm{S}_{11}$ & 0.0860 & wag & $40 \mathrm{GHz}$ \\
\hline $\mathrm{S}_{11}$ & 0.0836 & woag & $40 \mathrm{GHz}$ \\
\hline$Z_{11}$ & 51.62 & wag & $1 \mathrm{GHz}$ \\
\hline$Z_{11}$ & 53.91 & wag & $20 \mathrm{GHz}$ \\
\hline$Z_{11}$ & 47.29 & wag & $40 \mathrm{GHz}$ \\
\hline$Z_{11}$ & 57.43 & woag & $40 \mathrm{GHz}$ \\
\hline
\end{tabular}

D) Due to the low power level used in such measurements, normally recommended as around 1 to $3 \mathrm{~mW}$, the thermostatic case must provide a microcalorimeter kernel temperature stability of only a few $\mathrm{mK}$.

\subsection{INSTRUMENTATION SETUP AND THE MEASUREMENT PROCESS}

As Figure 5 below shows, the instrumentation setup consists of a thermostat implemented with a nested structure of active and passive thermal shields, two RF generators, two audio frequency (AF) current generators, two DC millivoltmeters, and two DC nanovoltmeters. Basically, this setup duplicates (if these instruments are not dual-channel models) the number of the instruments that are normally requested for running the classic coaxial microcalorimeter [1], [2], [4].

A switching box allows for supplying both the loads and dummy loads with correct RF/AF power levels according to the sequences requested for both the microcalorimeter calibration and power sensor calibration.

The dedicated computer controls the temperature inside the thermostat by means of Peltier elements, whereas a second computer performs the operations and the controls requested during all the measurement processes.

The measurement procedure starts when the microcalorimeter thermal loads are in thermal equilibrium, a status detected by the measured temperatures. The first phase consists of the characterization of the sensors pairing in three conditions: the same AF reference power (REF), the same sensor output voltage, and the same sensor temperatures (last calculated or measured at the connector reference plane). This data allows for the calculation of three ratios that are necessary for the next measurement and computing phases.
In the second phase, channel $\mathrm{A}$ is fed with an RF power level that produces the same microcalorimeter thermocouple output voltage as the AF REF simultaneously with a second RF source that is of the same frequency but with half the power level and is applied to the thermal reference line of channel A. This operation allows for extraction from the temperature response of the feeding line contribution due to its transmission losses. Finally, the effective efficiency is found using the ratio between the $\mathrm{AF}$ and RF thermopile output voltages for each test frequency.

Another algorithm can be applied in order to achieve thermopile balance. It can eliminate thermopile nonlinearity, but the operation does increase measurement time.

In [15], this main parameter of the thermoelectric power sensors, the effective efficiency, is obtained by a simple formula that uses the ratio between temperature values:

$$
\eta_{e}=\frac{e_{2}}{e_{1}-e_{1 S C}},
$$

where $e_{1}, e_{1 \mathrm{sc}}$, and $e_{2}$ are the thermopile responses in three situations realized in two steps: with the RF test power at the sensor input $\left(e_{1}\right)$, with the reference $\mathrm{AF}$ - power $\left(e_{2}\right)$, and with half of the RF test power. The sensor input is in short-circuit condition $\left(e_{1 s c}\right)$.

With the new proposed two-channel microcalorimeter, all these three quantities are available at the same time; moreover, no changes in the thermodynamic equilibrium of the system are provoked. The effective efficiency can be directly computed with simple formulas, like Equations (3) or (4) below, which are equivalent and basically have the same form. This effect is related to the introduction of the additional thermal reference lines. The formulae contain the thermopiles' output voltages ratio or the power sensors' output voltages ratio as well as the ratio of the two coefficients that define the pairing of the sensors and the balance in the feeding paths:

$\eta_{\text {eff } A}=\left.\frac{\left.P_{\text {in } S A}\right|_{R E F}}{\left.P_{\text {in } S A}\right|_{R F}}\right|_{E_{A}=c t .}=\frac{\left.e_{S B-D B}\right|_{R E F, E_{B}=E_{A}}}{\left.e_{S A-D A}\right|_{R F, E_{A}}} \frac{K_{S_{B-A}}}{K_{1_{B-A}}}$ and

$\eta_{e f f_{A}}=\frac{\left.E_{B}\right|_{R E F, E_{B}=\left.E_{A}\right|_{R F}}}{\left.E_{B}\right|_{R E F, e_{S B-D B}=\left.e_{S A-D A}\right|_{R F}}} \frac{K_{S_{B-A}}}{K_{1_{B-A}}}$,

where:

- $\eta_{\text {eff }}$ is the desired quantity, [1], [4], [5];

- $P_{\text {in } S A}$ is the input power dissipated in the power sensor that is being tested, [1], [4];

- $e_{S A-D A}, e_{S B-D B}$ are the thermopiles' differential output voltages;

- $E_{A}, E_{B}$ are the thermoelectric power sensors' output voltages;

- $K_{\mathrm{B}_{B-4}}$ defines the balance in the insulating lines in the thermal transmission coefficients [1], [5], [6], and [11]; and

- $K_{S_{B-4}}$ refers to the pairing of the power sensors in the power conversion sensitivity [9].

These two coefficients should be considered as being unitary as a consequence of two actions: power sensor pairing, $E_{A}=E_{B}$ for the same REF, and a balance in the thermopiles' differential output voltages, $e_{S A-D A}=e_{S B-D B}$, by applying a similar technique to that which is presented in Figure 3. Both Equations (3) and (4), therefore become ideal:

$\eta_{e f f_{A}}=\frac{\left.e_{S B-D B}\right|_{R E F, E_{B}=E_{A}}}{\left.e_{S A-D A}\right|_{R F, E_{A}}}$ and 


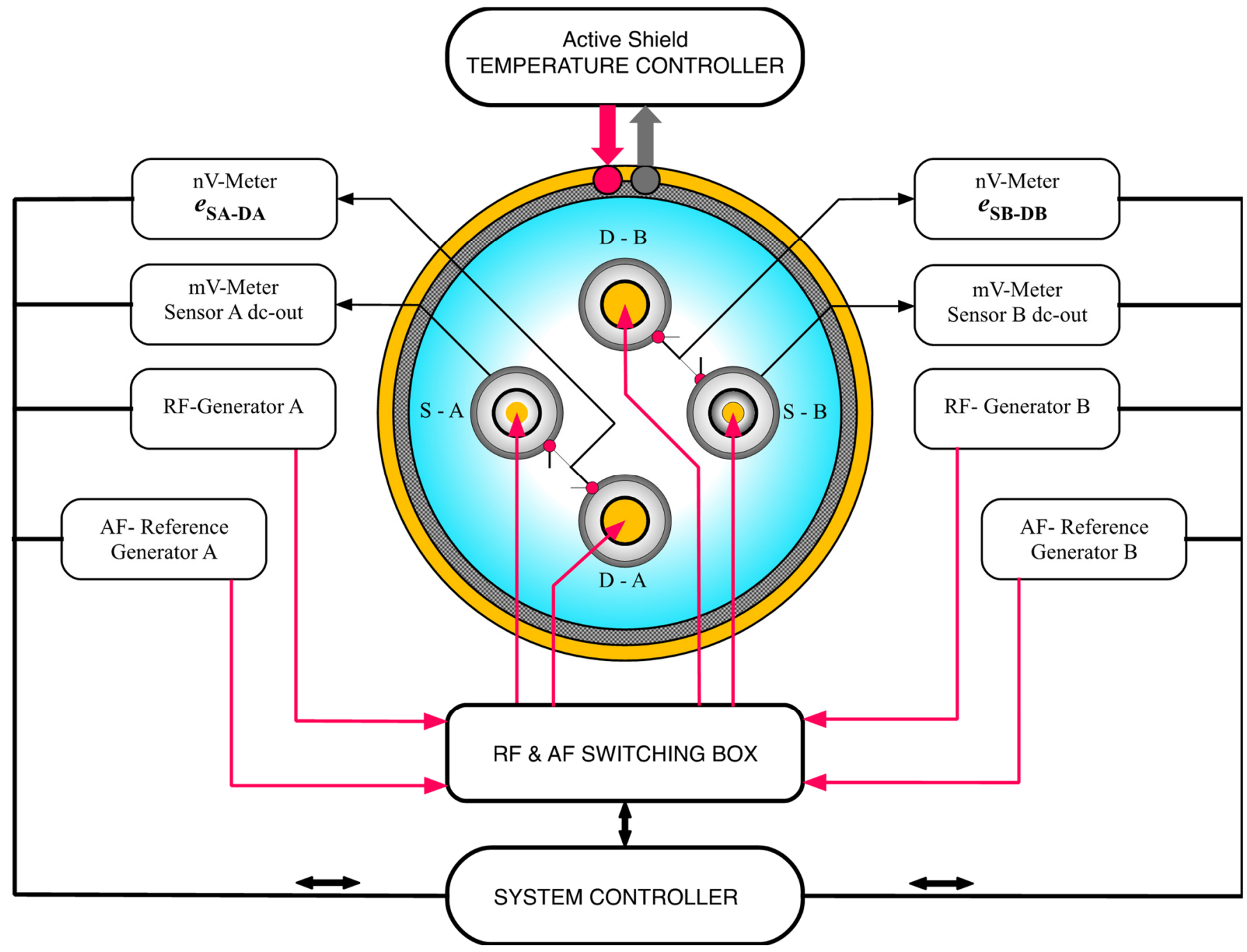

Figure 5. Scheme of the proposed instrumentation setup

$$
\eta_{e f f_{A}}=\frac{\left.E_{B}\right|_{R E F, E_{B}=\left.E_{A}\right|_{R F}}}{\left.E_{B}\right|_{R E F, e_{S B-D B}=\left.e_{S A-D A}\right|_{R F}}} .
$$

However, only (3) or (4) are considered in the uncertainty budget estimation. In this manner, we can take into account the imbalances and the imperfect pairing, regardless of the nonlinearity of the thermopiles [19]. If this nonlinearity affects the compensation of the line losses, the changes that may arise due to this effect must be estimated [20] and cancelled by adjusting the summation network, as shown in Figure 3. A simplified evaluation of the overall relative uncertainty leads to the next formulae:

$$
\begin{aligned}
& u_{\eta_{\%}}=\sqrt{\left(u_{e_{S A-D A}}\right)^{2}+\left(u_{e_{S B-D B}}\right)^{2}+\left(u_{K_{S}}\right)^{2}+\left(u_{K_{1}}\right)^{2}} \text { and } \\
& u_{\eta_{\%}}=\sqrt{\left(u_{E_{A}}\right)^{2}+\left(u_{E_{B}}\right)^{2}+\left(u_{K_{S}}\right)^{2}+\left(u_{K_{1}}\right)^{2}} .
\end{aligned}
$$

If we suppose that the four relative terms in Equation (7) and Equation (6) are under $0.1 \%$, an overall uncertainty of $0.2 \%$ is expected.

Another issue concerning the correctness of the algorithm measurements is represented by the correlated power leveling of both RF generators. Because the short circuit in the thermal reference is not perfect $\left(\Gamma_{\mathrm{SC}} \neq 1\right)$, the power ratio of the $\mathrm{RF}$ power inputs must be:

$$
\begin{aligned}
& P i_{I L_{D-4}}\left(1-\left|S_{21}\right|^{2}\right)\left(1+\left|\Gamma_{S C}\right|^{2}\left|S_{21}\right|^{2}\right)=P i_{I L_{S-4}}\left(1-\left|S_{21}\right|^{2}\right) \\
& \Rightarrow \frac{P i_{I L_{D-4}}}{P i_{I L_{S-4}}}=\frac{1}{1+\left|\Gamma_{S C}\right|^{2}\left|S_{21}\right|^{2}}>-3 d B
\end{aligned}
$$

where:

- $P i_{I L_{S-A}}$ and $P i_{I L_{D-A}}$ are the incident powers at the insulated lines' input for the sensor that is being tested and its thermal reference, respectively; and

- $S_{21}$ is the paired lines' forward transmission coefficient.

If Equation (9) is used in the RF power correlation, a degree of imbalance exists, however, due to the limits of the leveling loops. The leveling of RF power that is better than $0.02 \mathrm{~dB}$ and a power separation coefficient $k$ that is smaller than 0.1 lead to $0.05 \%$ uncertainty due to the imperfect compensation of the insulating line losses. This value does not have the effect of changing the estimated overall uncertainty.

At the end of the measurements according to the frequencies list, the whole process must be reiterated according to the computing requirements for calculating uncertainty and the number of degrees of freedom. A successful finish, proven by the same behaviors in the first phase of the measurement, allow for the immediate start of the measurements for the channel $\mathrm{B}$ sensor. The equations are, of course, similar to Equation pairs (3) and (5) or (4) and (6). 
The measurement time required for two paired RF power sensors and for the same number of degrees of freedom is half of the measurement time that is used in the actual microcalorimeter for a single sensor, which is, at least, 18 hours/six repeated measurements at every frequency test point.

\section{CONCLUSION}

The doubling of the channels in the classic twin microcalorimeter seems to be a better way of solving the problems still inherent in this architecture. Such a microcalorimeter and the new measurement methods and algorithms that can be applied solve the problems mentioned in the other research papers that have been dedicated to thermoelectric RF power standards [4-6, 11, 16-19]. The complications arising due to the realization of the true dualchannel RF microcalorimeter are mitigated by calibrating two paired sensors in a single measurement step and by involving coherently acquired data in the computation. In comparison with the methods described in the relevant literature until now [1-6, 8, 11, 15-21], this proposal offers key advantages: a much shorter measurement time, calculus that is based on two completely correlated datasets, and feeding line losses in each measurement moment.

\section{REFERENCES}

[1] A. E. Fantom, Radiofrequency and Microwave Power Measurement, Peter Peregrinus Ltd, London, 1990, ISBN 086341-120-7.

[2] F. R. Clague, Microcalorimeter for $7 \mathrm{~mm}$ coaxial transmission line, NIST Technical Note 1358, 1993.

[3] E. F. Glenn, Microwave Circuit Theory and Foundations of Microwave Metrology, Peter Peregrinus Ltd., London 1992, ISBN 0-86341-287-4.

[4] L. Brunetti, E. Vremera, A new microcalorimeter for measurements in 3.5-mm coaxial line, IEEE Transactions on Instrumentation and Measurement 52(2) (2003) pp. 320-323.

[5] L. Brunetti, L. Oberto, M. Sellone, E. Vremera, Latest determination of a coaxial microcalorimeter calibration factor, Measurement Science and Technology 22 (2011) pp. 025101-1025101-6

[6] E. Vremera, L. Brunetti, L. Oberto, M. Sellone, Power sensor calibration by implementing true-twin microcalorimeter, IEEE Trans. Instr. Meas. 60(7) (2011) pp. 2335-2340.
[7] E. Vremera, L. Brunetti, "Primary RF-Power Standard Realization in a Single-step Measurement Process", Proc. of 22 nd $I M E K O$ TC4 Int. Symposium and 20th Int. Workshop on ADC Modelling and Testing, Sept. 14-15, 2017, Iasi, Romania, pp. 424-428.

[8] J. Y. Kwon, D. J. Lee, Adiabatic design for a coaxial transmission line, IEEE Transactions on Instrumentation and Measurement 63(7) (2014) pp. 1760-1768.

[9] G. C. M. Meijer, A.W. Herwaarden (editors), Thermal Sensors, Inst. of Physics Publishing, Bristol, 1994, ISBN 0-7503-0220-8.

[10] T. Hatakeyama, F. X. Quinn (editors), Thermal Analysis: Fundamentals and Applications to Polymer Science, John Wiley \& Sons Ltd., London 1999, ISBN 978-0-471-98362-0.

[11] E. Vremera, L. Brunetti, Design of $2.92 \mathrm{~mm}$ coaxial adiabatic lines for quasi-ideal twin microcalorimeter, IEEE Transactions on Instrumentation and Measurement 61(6) (2012) pp. 1692-1702.

[12] C. B. Carter, M. G. Norton, Ceramic Materials: Science and Engineering, Springer, New York, 2007, ISBN 978-0-387-462714.

[13] J. Ma, H. H. Hng, High thermal conductivity ceramic layered system substrates for microelectronic applications, Journal of Materials Science: Materials in Electronics 13 (2002) pp. 461-464.

[14] S. L. Shindé, J. Goela (editors), High Thermal Conductivity Materials, Springer, New York 2006, ISBN 978-0-387-22021-5.

[15] L. Brunetti, L. Oberto, E. Vremera, Thermoelectric sensors as microcalorimeter load, IEEE Transactions on Instrumentation and Measurement 56(6) (2007) pp. 2220-2224.

[16] E. Vremera, L. Brunetti, L. Oberto, M. Sellone, Alternative procedures in realizing of the high frequency power standards with microcalorimeter and thermoelectric power sensors, Measurement 42(2) (2009) pp. 269-276.

[17] J. Y. Kwon, T. W. Kang, J. H. Kim, J. S. Kang, Development of a $3.5 \mathrm{~mm}$ coaxial microcalorimeter for RF and microwave power standards at KRISS, IEEE Transactions on Instrumentation and Measurement 60(7) (2011) pp. 2609-2614.

[18] L. Brunetti, L. Oberto, M. Sellone, E. Vremera, Comparison between thermoelectric and bolometric microwave power sensors, IEEE Transactions on Instrumentation and Measurement 62(6) (2013) pp. 1710-1715.

[19] L. Brunetti, E. Monticone, Thermopile linearity errors in the HFcalorimeter method, Measurement 8(4) (1990) pp. 146-152.

[20] J. Y. Kwon, T. W. Kang, N. W. Kang, V-band waveguide microcalorimeter for millimeter-wave power standards, IEEE Trans. Instr. Meas. 66(6) (2017) pp. 1598-1604.

[21] L. Oberto, L. Brunetti, RF/MW power standard realization without unitary efficiency assumption at DC/LF, IEEE Trans. Instr. Meas. 67(4) (2018) pp. 925-929 